\title{
Skorohod Integral at Vacuum State on Guichardet-Fock Spaces
}

\author{
Jihong Zhang1, Yongjun Li' ${ }^{1}$, Xiaochun Sun ${ }^{2}$ \\ ${ }^{1}$ School of Mathematics, Lanzhou City University, Lanzhou, China \\ ${ }^{2}$ College of Mathematics and Statistics, Northwest Normal University, Lanzhou, China \\ Email: zhjhzhangjihong@163.com
}

Received 23 May 2016; accepted 16 July 2016; published 19 July 2016

\begin{abstract}
In this paper, we define expectation of $f \in F$, i.e. $E(f)=f(\varnothing)$, according to Wiener-Ito-Segal isomorphic relation between Guichardet-Fock space $F$ and Wienerspace $W$. Meanwhile, we derive a formula for the expectation of random Hermite polynomial in Skorohod integral on GuichardetFock spaces. In particular, we prove that the anticipative Girsanov identities under the condition $E\left(H_{n}\left(\delta(x),\|x\|^{2}\right)\right)=0, n \geq 1$ on Guichardet-Fock spaces.
\end{abstract}

\section{Keywords}

\section{Moment Identities, Girsanov Identities, Hermitpolynomial, Skorohod Integral, Guichardet-Fock} Spaces

\section{Introduction}

The quantum stochastic calculus developed by Hudson and Parthasarathy [1] is essentially a noncommutative extension of classical Ito stochastic calculus. In thistheory, annihilation, creation, and number operator processes in boson Fock space play the role of "quantum noises" [2] [3], which are in continuous time. In 2002, Attal [4] discussed and extended quantum stochastic calculus by means of the Skorohod integral of anticipation processes and the related gradient operator on Guichardet-Fock spaces. Usually, Fock spaces as the models of the Particle Systems are widely used in quantumphysics. Meanwhile, vacuum states described by empty set on Guichardet-Fockspaces play very important role at quantum physics.

Recently Privault [5] [6] developed a Malliavin-type theory of stochastic calculus on Wiener spaces and showed its several interesting applications. In his article, Privault surveyed the moment identities for Skorohod integral and derived a formula for the expectation of random Hermit polynomials in Skorohod integral on Wiener spaces. It is well known that Guichardet-Fock space $F$ and Wiener space W are Wiener-Ito-Segal isomorphic. Motivated by the above, we would like to study the expectation of random Hermit polynomials in Skorohod integral on Guichardet-Fock spaces. However, how to define the expectation on Guichardet-Fock spaces is the primary problem.

In this argument, we define expectation of $f \in F$ according to isomorphic relation, i.e. $E(f)=f(\varnothing)$.

Meanwhile, we prove a moment identity for the Skorohod integrals and derive a formula for the expectation 
of random Hermite polynomial in Skorohod integral on Guichardet-Fock spaces. Particularly, under the condition $E\left(H_{n}\left(\delta(x),\|x\|^{2}\right)\right)=0, n \geq 1$, we prove the anticipative Girsanov identities on Guichardet-Fock spaces.

This paper is organized as follows. Section 2, we fix some necessarynotations and recall main notions and facts about Skorohod integral in Guichardet-Fock spaces. Section 3 and Section 4 state our main results.

\section{Notations}

In this section, we fix some necessary notations and recall mainnotions in Guichardet-Fock spaces. For detail formulation of Skorohod integrals, we refer reader to [4].

Let $R_{+}$be the set of all nonnegative real numbers and $\Gamma$ the finite power set of $R_{+}$, namely

$$
\Gamma:=\left\{\sigma \mid \sigma \subset R_{+}, \#<\infty\right\},
$$

where denotes the cardinality of $\sigma$ as a set. Particularly, let $\varnothing \in \Gamma^{(0)}$ be an atom of measure 1 . We denote by $L^{2}(\Gamma)$ the usual space of square integral real-valued functions on $\Gamma$.

Fixing a complex separable Hilbert space $\eta$, Guichardet-Fock space tensor product $\eta \otimes L^{2}(\Gamma)$, which we identify with the space of square-integrable functions $L^{2}(\Gamma ; \eta)$, is denoted by $F$.

For a Hilbert space-valued map $x: \Gamma \times R_{+} \rightarrow \eta$, let

$$
\delta(x): \sigma \mapsto \sum_{s \in \sigma} x_{s}(\sigma \backslash s)
$$

denotes the Skorohod integral operator. For a vector space-valued map $f: \Gamma \rightarrow V$, let $\nabla f$ and $D f$ be the maps $\Gamma \times R_{+} \rightarrow V$ given by

$$
\nabla f(\omega, s)=f(\omega \bigcup s), D f(\omega, s)=\mathbf{1}_{\{\omega<s\}} f(\omega \bigcup s)
$$

respectively denote the stochastic gradient operator of $f$ and the adapted gradient operator of $f$. Moreover, we write $\operatorname{Dom} \nabla$ for the domain of the stochastic gradient as anunbounded Hilbert apace operator:

$$
\operatorname{Dom} \nabla:=\left\{f \in F: \nabla f \in L^{2}\left(\Gamma \times R_{+} ; \eta\right)\right\} .
$$

Definition 2.1 The value of $f \in F$ at empty set is called the expectation of $f$ on Guichardet-Fock space and is denoted by $E(f)$.i.e. $E(f)=f(\varnothing)$

Definition 2.2 For the map $x: \Gamma \times R_{+} \rightarrow \eta$, the value of Skorohod integral $\delta(x)$ at empty set is called the expectation of $\delta(x)$ on Guichardet-Fock space and is denoted by $E(\delta(x))$ i.e. $E(\delta(x))=\delta(x)(\varnothing)$.

Lemma 2.1 Let $x$ be a map $\Gamma \times R_{+} \rightarrow \eta$, if $x$ is square integrable and the function $(\omega, s, t) \rightarrow\left\langle x_{s}(\omega \bigcup t), x_{t}(\omega \bigcup s)\right\rangle$ is integrable, then $x \in \operatorname{Dom} \delta$ and

$$
\|\delta(x)\|^{2}=\int\|x\|^{2} d s+\iiint\left\langle x_{s}(\omega \bigcup t), x_{t}(\omega \bigcup s)\right\rangle d \omega d t d s,
$$

we denote

$$
\begin{aligned}
\operatorname{trace}(D x)^{2} & =\left\langle\nabla x, \nabla^{*} x\right\rangle \\
& =\int_{0}^{\infty} \int_{0}^{\infty}\left\langle\nabla_{t} x_{s}, \nabla_{s} x_{t}\right\rangle d t d s \\
& =\int_{0}^{\infty} \int_{0}^{\infty}\left\langle x_{s}(\omega \cup t), x_{t}(\omega \cup s)\right\rangle d t d s .
\end{aligned}
$$

Lemma 2.2 Let $f \in F$ and let $x: \Gamma \times R_{+} \rightarrow \eta$ be Skorohod integrable, if the map

$$
(\omega, s) \mapsto\left\langle x_{s}(\omega), f(\omega \cup s)\right\rangle
$$

is integrable, then

$$
\langle\delta(x), f\rangle=\iint\left\langle x_{\omega}, \nabla_{s} f(\omega)\right\rangle d \omega d s .
$$

Lemma 2.3 Let $x: \Gamma \times R_{+} \rightarrow \eta$ be measurable. For a.a.t, we have

$$
D_{t} \delta(x)=\delta_{0}^{t}\left(D_{t} x\right)+P_{t} x_{t},
$$

where $P_{t} x_{t}=\mathbf{1}_{\Gamma_{t}} x_{t}, \Gamma_{t}:=\{\omega \in \Gamma: \omega \subset[0, t[\}$.

Theorem 2.1 For any $n \geq 1$ and $x \in F$, we have 


$$
E\left(\delta(x)^{n+1}\right)=\sum_{k=1}^{n} \frac{n !}{(n-k) !} E\left[\delta(x)^{n-k}\left(\left\langle(\nabla x)^{k-1} x, x\right\rangle+\operatorname{trace}(\nabla x)^{k+1}+\sum_{i=2}^{k} \frac{1}{i}\left\langle(\nabla x)^{k-i} x, \nabla \operatorname{trace}(\nabla x)^{i}\right\rangle\right)\right],
$$

where

$$
\operatorname{trace}(\nabla x)^{k+1}=\int_{0}^{\infty} \cdots \int_{0}^{\infty}\left\langle\nabla_{t_{k-1}}^{*} x_{t_{k}}, \nabla_{t_{k-2}} x_{t_{k-1}} \cdots \nabla_{t_{0}} x_{t_{1}} \nabla_{t_{k}} x_{t_{0}}\right\rangle d t_{0} \cdots d t_{k} \text {. }
$$

Lemma 2.4 Let $n \geq 1$ and $x \in F$. Then for all $1 \leq k \leq n$ we have

$$
\begin{aligned}
& E\left(\delta(x)^{n-k}\left\langle(\nabla x)^{k-1} x, \nabla \delta(x)\right\rangle\right)(\varnothing)-(n-k)\left(\delta(x)^{n-k-1}\left\langle(\nabla x)^{k} x, \nabla \delta(x)\right\rangle\right) \\
& =E\left[\delta(x)^{n-k}\left(\left\langle(\nabla x)^{k-1} x, x\right\rangle+\operatorname{trace}(\nabla x)^{k+1}+\sum_{i=2}^{k} \frac{1}{i}\left\langle(\nabla x)^{k-i} x, \nabla \operatorname{trace}(\nabla x)^{i}\right\rangle\right)\right] .
\end{aligned}
$$

\section{Random Hermit Polynomials}

In Theorem 3.1 below, we compute the expectation of the random Hermit polynomial $E\left(H_{n}\left(\delta(x),\|x\|^{2}\right)\right)$ with respect to the Skorohod integral $\delta(x), n \geq 1$. This result will be applied in Section 4 to anticipate Girsanov identities on Guichardet-Fock spaces.

Theorem 3.1 For any $n \geq 0$ and $x: \Gamma \times R_{+} \rightarrow \eta$, we have

$$
E\left(H_{n+1}\left(\delta(x),\|x\|^{2}\right)\right)=\sum_{l=0}^{n-1} \frac{n !}{l !} E\left[\delta(x)^{l} \sum_{0 \leq 2 k \leq n-1-l} \frac{(-1)^{k}}{k !} \frac{\|x\|^{2 k}}{2^{k}} ? \nabla x \nabla \quad \nabla x\left(\left(^{2 k-}\right)^{-1} x\right\rangle\right.
$$

Especially, for $x$ and

$$
\left\langle\nabla x, \nabla\left((\nabla x)^{k} x\right)\right\rangle=0,0 \leq k \leq n-2,
$$

then we have

$$
E\left(H_{n+1}\left(\delta(x),\|x\|^{2}\right)\right)=0, \quad n \geq 1 .
$$

Proof We divide two steps to prove the stability result.

Step 1 . We first prove that for any $n \geq 1$,

$$
\begin{aligned}
& E\left(H_{n+1}\left(\delta(x),\|x\|^{2}\right)\right)=\sum_{0 \leq 2 k \leq n-1}(-1)^{k} \frac{n !}{k ! 2^{k}(n-2 k-1) !} \cdot E\left(\delta(x)^{n-2 k-1}\langle x, x\rangle\langle x, \delta(\nabla x)\rangle\right)+ \\
& \sum_{1 \leq 2 k \leq n}(-1)^{k} \frac{n !}{k ! 2^{k}(n-2 k) !} \cdot E\left(\delta(x)^{n-2 k}\left\langle x, \nabla\langle x, x\rangle^{k}\right\rangle\right),
\end{aligned}
$$

For $f \in F$ and $l, k \geq 1$, we have

$$
\begin{aligned}
& \delta(x)^{l+1}=\frac{l+2 k+1}{2 k} f \delta(x)^{l+1}-\frac{l+1}{2 k} f \delta(x)^{l+1} \\
& =\frac{l+2 k+1}{2 k} f \delta(x)^{l+1}-\frac{l+1}{2 k}\left\langle x, \nabla\left(\delta(x)^{l} f\right)\right\rangle \\
& =\frac{l+2 k+1}{2 k} f \delta(x)^{l+1}-\frac{l(l+1)}{2 k} f \delta(x)^{l-1}\langle x, \nabla \delta(x)\rangle-\frac{l+1}{2 k} \delta(x)^{l}\langle x, \nabla f\rangle \\
& =\frac{l+2 k+1}{2 k} f \delta(x)^{l+1}-\frac{l(l+1)}{2 k} f \delta(x)^{l-1}\langle x, x\rangle-\frac{l(l+1)}{2 k} f \delta(x)^{l-1}\langle x, \delta(\nabla x)\rangle-\frac{l+1}{2 k} \delta(x)^{l}\langle x, \nabla f\rangle,
\end{aligned}
$$

replace 1 above with $n-2 k$, we have

$$
\begin{aligned}
& \delta(x)^{n-2 k+1}+\frac{(n-2 k)(n-2 k+1)}{2 k} f \delta(x)^{n-2 k-1}\langle x, x\rangle \\
& =\frac{n+1}{2 k} f \delta(x)^{n-2 k+1}-\frac{(n-2 k)(n-2 k+1)}{2 k} f \delta(x)^{n-2 k-1}\langle x, \delta(\nabla x)\rangle-\frac{n-2 k+1}{2 k} \delta(x)^{n-2 k}\langle x, \nabla f\rangle,
\end{aligned}
$$

Hence, taking $f=\langle x, x\rangle^{k}$, we get 


$$
\begin{aligned}
& E\left(\delta(x)^{n+1}\right)=E\left(\left\langle x, \nabla \delta(x)^{n}\right\rangle\right)=E\left(n \delta(x)^{n-1}\langle x, \nabla \delta(x)\rangle\right) \\
& =E\left(n \delta(x)^{n-1}\langle x, x\rangle\right)+E\left(n \delta(x)^{n-1}\langle x, \delta(\nabla x)\rangle\right) \\
& =E\left(n \delta(x)^{n-1}\langle x, \delta(\nabla x)\rangle\right)-\sum_{1 \leq 2 k \leq n+1}(-1)^{k} \\
& \times \frac{n !}{(k-1) ! 2^{k-1}(n+1-2 k) !} E\left(\delta(x)^{n-2 k+1}\langle x, x\rangle^{k}\right) \\
& +\frac{(n-2 k+1)(n-2 k)}{2 k} E\left(\delta(x)^{n-2 k-1}\langle x, x\rangle^{k+1}\right) \\
& =E\left(n \delta(x)^{n-1}\langle x, \delta(\nabla x)\rangle\right)-\sum_{1 \leq 2 k \leq n+1}(-1)^{k} \\
& \times \frac{n !}{(k-1) ! 2^{k-1}(n+1-2 k) !} E\left(\frac{n+1}{2 k} \delta(x)^{n-2 k+1}\langle x, x\rangle^{k}\right) \\
& -\frac{(n-2 k)(n-2 k+1)}{2 k} E\left(\delta(x)^{n-2 k-1}\langle x, x\rangle^{k}\langle x, \delta(\nabla x)\rangle\right) \\
& -\frac{n-2 k+1}{2 k} E\left(\delta(x)^{n-2 k}\left\langle x, \nabla\langle x, x\rangle^{k}\right\rangle\right) \\
& =-\sum_{1 \leq 2 k \leq n+1}(-1)^{k} \frac{(n+1) !}{k ! 2^{k}(n+1-2 k) !} E\left(\delta(x)^{n-2 k+1}\langle x, x\rangle^{k}\right) \\
& +\sum_{0 \leq 2 k \leq n-1}(-1)^{k} \frac{n !}{k ! 2^{k}(n-1-2 k) !} E\left(\delta(x)^{n-2 k-1}\langle x, x\rangle^{k}\langle x, \delta(\nabla x)\rangle\right) \\
& +\sum_{1 \leq 2 k \leq n}(-1)^{k} \frac{n !}{k ! 2^{k}(n-2 k) !} E\left(\delta(x)^{n-2 k}\left\langle x, \nabla\langle x, x\rangle^{k}\right\rangle\right) .
\end{aligned}
$$

Step 2. For $f \in F$, and $0 \leq i \leq l$, we have

$$
\begin{aligned}
& E\left(f \delta(x)^{l}\left\langle(\nabla x)^{i} x, \delta(\nabla x)\right\rangle\right)-l E\left(f \delta(x)^{l-1}\left\langle(\nabla x)^{i+1} x, \delta(\nabla x)\right\rangle\right) \\
&= E\left(\left\langle\nabla x, \nabla\left(f \delta(x)^{l}(\nabla x)^{i} x\right)\right\rangle\right)-l E\left(f \delta(x)^{l-1}\left\langle(\nabla x)^{i+1} x, \delta(\nabla x)\right\rangle\right) \\
&= l E\left(f \delta(x)^{l-1}\left\langle\nabla x,(\nabla x)^{i} x \otimes \nabla \delta(x)\right\rangle\right)-l E\left(f \delta(x)^{l-1}\left\langle(\nabla x)^{i+1} x, \delta(\nabla x)\right\rangle\right) \\
&+E\left(\delta(x)^{l}\left\langle\nabla x, \nabla\left(f(\nabla x)^{i} x\right)\right\rangle\right) \\
&= l E\left(f \delta(x)^{l-1}\left\langle\nabla x,(\nabla x)^{i} x \otimes x\right\rangle\right)+l E\left(f \delta(x)^{l-1}\left\langle\nabla x,(\nabla x)^{i} x \otimes \delta(\nabla x)\right\rangle\right) \\
&- l E\left(f \delta(x)^{l-1}\left\langle(\nabla x)^{i+1} x, \delta(\nabla x)\right\rangle\right)+E\left(\delta(x)^{l}\left\langle\nabla x, \nabla\left(f(\nabla x)^{i} x\right)\right\rangle\right) \\
&= l E\left(f \delta(x)^{l-1}\left\langle(\nabla x)^{i+1} x, x\right\rangle\right)+E\left(\delta(x)^{l}\left\langle(\nabla x)^{i+1} x, \nabla f\right\rangle\right)+E\left(f \delta(x)^{l}\left\langle\nabla x, \nabla\left((\nabla x)^{i} x\right)\right\rangle\right) .
\end{aligned}
$$

Hence, replacing 1 above with $l-i$, we get

$$
\begin{aligned}
& E\left(f \delta(x)^{l}\langle x, \delta(\nabla x)\rangle\right) \\
& =l ! E\left(f\left\langle(\nabla x)^{l} x, \delta(\nabla x)\right\rangle\right)+l E\left(f \delta(x)^{l-1}\langle x, \delta(\nabla x)\rangle\right) \\
& =l ! E\left(f\left\langle(\nabla x)^{l} x, \delta(\nabla x)\right\rangle\right)+\sum_{i=0}^{l-1} \frac{l !}{(l-i) !} E\left(f \delta(x)^{l-i}\left\langle(\nabla x)^{i} x, \delta(\nabla x)\right\rangle\right) \\
& -(l-i) E\left(f \delta(x)^{l-i-1}\left\langle(\nabla x)^{i+1} x, \delta(\nabla x)\right\rangle\right) \\
& =l ! E\left(\left\langle(\nabla x)^{l+1} x, \nabla f\right\rangle\right)+\sum_{i=0}^{l-1} \frac{l !}{(l-i-1) !} ? E f \delta\left((x)^{n-2 k-1}\left\langle(\nabla x)^{i+1} x, x\right\rangle\right) \\
& +\sum_{i=1}^{l} \frac{l !}{l-i+1} E\left(\delta(x)^{l-i+1}\left\langle(\nabla x)^{i} x, \nabla f\right\rangle\right)+\sum_{i=0}^{l} \frac{l !}{(l-i) !} E\left(f \delta(x)^{l-i}\left\langle\nabla x, \nabla\left((\nabla x)^{i} x\right)\right\rangle\right),
\end{aligned}
$$

thus letting $f=\langle x, x\rangle^{k}$ and $l=n-2 k-1$ above, and use (2.3) in step 1, we get 


$$
\begin{aligned}
& E\left(H_{n+1}\left(\delta(x),\|x\|^{2}\right)\right) \\
& =\sum_{0 \leq 2 k \leq n-1}(-1)^{k} \frac{n !}{k ! 2^{k}(n-2 k-1) !} E\left(\delta(x)^{n-2 k-1}\langle x, x\rangle^{k}\langle x, \delta(\nabla x)\rangle\right) \\
& +\sum_{1 \leq 2 k \leq n}(-1)^{k} \frac{n !}{k ! 2^{k}(n-2 k) !} E\left(\delta(x)^{n-2 k}\left\langle x, \nabla\langle x, x\rangle^{k}\right\rangle\right) \\
& =\sum_{0 \leq 2 k \leq n}(-1)^{k} \frac{n !}{k ! 2^{k}} E\left(\left\langle(\nabla x)^{n-2 k} x, \nabla\langle x, x\rangle^{k}\right\rangle\right) \\
& +\sum_{0 \leq 2 k \leq n-1} \frac{(-1)^{k}}{k ! 2^{k}} \sum_{i=0}^{n-2 k-2} \frac{n !}{(n-2(k+1)-i) !} E\left(\langle x, x\rangle^{k} \delta(x)^{n-2(k+1)-i}\left\langle(\nabla x)^{i+1} x, x\right\rangle\right) \\
& +\sum_{0 \leq 2 k \leq n-1} \frac{(-1)^{k}}{k ! 2^{k}} \sum_{i=1}^{n-2 k-1} \frac{n !}{(n-2 k-i) !} E\left(\delta(x)^{n-2 k-i}\left\langle(\nabla x)^{i} x, \nabla\langle x, x\rangle^{k}\right\rangle\right) \\
& +\sum_{0 \leq 2 k \leq n-1} \frac{(-1)^{k}}{k ! 2^{k}} \sum_{i=0}^{n-2 k-1} \frac{n !}{(n-2 k-1-i) !} \cdot E\left(\langle x, x\rangle^{k} \delta(x)^{n-2 k-i-1}\left\langle\nabla x, \nabla\left((\nabla x)^{i} x\right)\right\rangle\right) \\
& +\sum_{0 \leq 2 k \leq n-1}(-1)^{k} \frac{n !}{k ! 2^{k}(n-2 k) !} E\left(\delta(x)^{n-2 k}\left\langle x, \nabla\langle x, x\rangle^{k}\right\rangle\right) \\
& =\sum_{0 \leq 2 k \leq n-1} \frac{(-1)^{k} n-2 k-1}{k ! 2^{k}} \sum_{i=0}^{n !} \frac{n !}{(n-2 k-1-i) !} E\left(\langle x, x\rangle^{k} \delta(x)^{n-2 k-i-1}\left\langle\nabla x, \nabla\left((\nabla x)^{i} x\right)\right\rangle\right) .
\end{aligned}
$$

\section{Girsanov Identities}

Corollary 4.1 Assume that $x: \Gamma \times R_{+} \rightarrow \eta$ with $E\left(e^{|\delta(x)|+\frac{1}{2}}\|x\|^{2}\right)<\infty$ and that $\nabla x$ holds (3.1). Then, we have

$$
E\left(\exp \left(\delta(x)-\frac{1}{2}\|x\|^{2}\right)\right)=1
$$

Proof We have

$$
\left|H_{n}\left(x, \sigma^{2}\right)\right| \leq \sum_{0 \leq 2 k \leq n} \frac{(-1)^{k}}{k ! 2^{k}} \frac{n !}{(n-2 k) !}|x|^{n-2 k}\left(-\sigma^{2}\right)^{k}=H_{n}\left(|x|,-\sigma^{2}\right),
$$

hence

$$
\sum_{n=0}^{\infty} \frac{1}{n !} E\left|H_{n}\left(\delta(x),\|x\|^{2}\right)\right| \leq \sum_{n=0}^{\infty} \frac{1}{n !} E\left(H_{n}\left(|\delta(x)|,-\|x\|^{2}\right)\right)=E\left(e^{|\delta(x)|+\frac{1}{2}\|x\|^{2}}\right)<\infty .
$$

By Theorem 3.1 and Fubini theorem, we have

$$
\begin{aligned}
& E\left(\exp \left(\delta(x)-\frac{1}{2}\|x\|^{2}\right)\right)=1+\sum_{n=0}^{\infty} \frac{1}{(n+1) !} E\left(H_{n}\left(|\delta(x)|,\|x\|^{2}\right)\right) \\
& =1+\sum_{n=0}^{\infty} \frac{1}{(n+1) !} E\left(H_{n+1}\left(|\delta(x)|,\|x\|^{2}\right)\right)=1 .
\end{aligned}
$$

This shows that $\|x\|$ is deterministic and $\nabla x$ holds (3.1), we have

$$
E\left(e^{\delta(x)}\right)=E\left(e^{\frac{1}{2}\|x\|^{2}}\right),
$$

i.e. $\delta(x)$ has a centered Gaussian distribution with variance $\|x\|^{2}$ on Guichardet-Fock spaces.

\section{Acknowledgements}

The authors are extremely grateful to the referees for their valuable comments and suggestions on improvement of the first version of the present paper. The authors are supported by National Natural Science Foundation of 
China (No. 11261027 and No. 11461061), supported by scientific research projects in Colleges and Universities in gansu province (No. 2015A-122) and supported by doctoral research start-up fund project of Lanzhou City Universities (No. LZCU-BS2015-02) and SRPNWNU (No. NWNU-LKQW-14-2).

\section{References}

[1] Hudson, R.L. and Parthasarathy, K.R. (1984) Quantum Ito’s Formula and Stochastic Evolutions. Communications in Mathematical Physics, 3, 301-323. http://dx.doi.org/10.1007/BF01258530

[2] Wang, C.S., Lu, Y.C. and Chai, H.F. (2011) An Alternative Approach to Privault's Discrete-Time Chaotic Calculus. Journal of Mathematical Analysis and Applications, 2, 643-654. http://dx.doi.org/10.1016/j.jmaa.2010.08.021

[3] Zhang, J.H., Wang, C.S. and Tian, L.N. (2015) Localization of Unbounded Operators on Guichardet Spaces. Journal of Applied Mathematics and Physics, 3, 792-796. http://dx.doi.org/10.4236/jamp.2015.37096

[4] Attal, S. and Lindsay, J.M. (2004) Quantum Stochastic Calculus with Maximal Operator Domains. The Annals of Probability, 32, 488-529. http://dx.doi.org/10.1214/aop/1078415843

[5] Privault, N. (2009) Moment Identities for Skorohod Integrals on the Wiener Space and Applications. Electronic Communications in Probability, 14, 116-121. http://dx.doi.org/10.1214/ECP.v14-1450

[6] Privault, N. (2010) Random Hermite Polynomials and Girsanov Identities on the Wiener Space. Infinite Dimensional Analysis, 13, 663-675.

\section{Submit or recommend next manuscript to SCIRP and we will provide best service for you:}

Accepting pre-submission inquiries through Email, Facebook, LinkedIn, Twitter, etc.

A wide selection of journals (inclusive of 9 subjects, more than 200 journals)

Providing 24-hour high-quality service

User-friendly online submission system

Fair and swift peer-review system

Efficient typesetting and proofreading procedure

Display of the result of downloads and visits, as well as the number of cited articles

Maximum dissemination of your research work

Submit your manuscript at: http://papersubmission.scirp.org/ 